\title{
The WASh Trial: water-assisted sigmoidoscopy in the English Bowel Scope Screening Programme: study protocol for a randomized multicenter trial
}

\section{(ㄷ)(우우}

Authors

Iosif Beintaris ${ }^{1}$, Shiran Esmaily¹, Brian P. Saunders², Colin J. Rees ${ }^{3}$, Christian Von Wagner ${ }^{4}$, Zacharias Tsiamoulos ${ }^{2}$, Zoe Hoare $^{5}$, Rachel Evans ${ }^{5}$, Seow Tien Yeo ${ }^{6}$, R. T. Edwards ${ }^{6}$, Tony Larkin ${ }^{7}$, Andrew Veitch ${ }^{8}$, Andrew Chilton ${ }^{9}$, Michael G. Bramble $^{10}$, Jill Deane ${ }^{1}$, Matthew D. Rutter ${ }^{1,11,12}$

Institutions

1 Department of Gastroenterology, University Hospital of North Tees, Stockton-On-Tees, UK

2 Wolfson Unit of Endoscopy, St Mark's Hospital, London, UK

3 Department of Gastroenterology, South Tyneside NHS Trust, South Shields UK

4 Research Department of Epidemiology and Public Health, University College London, UK

5 North Wales Organisation for Randomised Trials in Health, UK

6 Centre for Health Economics and Medicines Evaluation, Bangor University, UK

710 Tulip Close, Hartlepool

8 Department of Gastroenterology, New Cross Hospital, Wolverhampton, UK

9 Department of Gastroenterology, Kettering General Hospital NHS Foundation Trust, UK

10 Department of Gastroenterology, James Cook University Hospital, Middlesbrough, UK

11 School of Medicine Pharmacy and Health, Durham University, UK

12 Northern Institute for Cancer Research, Newcastle University, UK

submitted 10.10 .2018

accepted after revision 23.4.2019

\section{Bibliography}

DOI https://doi.org/10.1055/a-0953-1468 |

Endoscopy International Open 2019; 07: E1574-E1582

(c) Georg Thieme Verlag KG Stuttgart · New York

elSSN 2196-9736

Corresponding author

Matt Rutter, North Tees and Hartlepool NHS Foundation

Trust - Gastroenterology, Hardwick Rd, Hardwick Stocktonon-Tees TS24 9AH, United Kingdom of Great Britain and Northern Ireland
Fax: +01642 617617

Matt.Rutter@nth.nhs.uk

\section{ABSTRACT}

Background and study aims The English National Bowel Scope Screening Programme (BSSP) invites 55-year-olds for a one-off, unsedated flexible sigmoidoscopy (FSIG). Data from BSSP participant-reported experience studies shows 1 in 3 participants report moderate or severe discomfort. Water-assisted colonoscopy (WAS) may improve participants' comfort. The primary objective of this study is to ascertain if post-procedural participant-assessed pain is reduced in WAS compared with carbon dioxide $\left(\mathrm{CO}_{2}\right)$ insufflation, in invitees undergoing FSIG in BSSP.

Patients and methods This is a multicenter, prospective, randomized, two-arm, single-blinded trial designed to evaluate the performance of WAS versus $\mathrm{CO}_{2}$ insufflation in BSSP. Participants will be randomized to either $\mathrm{CO}_{2}$ or WAS and will be asked to rate pain post-procedure. Key procedure-related data will be analyzed, including adenoma detection rates (ADR) and degree of sigmoid looping. A costeffectiveness analysis of WAS versus $\mathrm{CO}_{2}$ and a discrete choice experiment exploring preferences of participants for attributes of sigmoidoscopy will also be performed.

Discussion This is the first trial in the United Kingdom (UK) to investigate the effects of WAS in a screening setting. If the trial shows WAS either reduces pain or increases ADR, this may result in a practice change to implement WAS in screening and non-screening endoscopic practice directly impacting on 256,000 people a year who will undergo BSSP FSIG by 2020 .

Trial funding came from National Institute for Health Research (NIHR) Research for Patient Benefit (RfPB) supported by the NIHR Clinical Research Network. The trial is actively recruiting. ID: 35866 ISRCTN: 81466870 


\section{Introduction}

Results from a large, multicenter, randomized controlled trial in the UK showed that screening people aged 55 to 64 years with a single flexible sigmoidoscopy (FSIG) reduced colorectal cancer (CRC) incidence and mortality by $33 \%$ and $43 \%$ respectively [1]. A subsequent pilot study performed in three English sites confirmed that FSIG screening of participants aged 55 years is both feasible and acceptable [2]. Based on this, Bowel Scope Screening Programme (BSSP) is being rolled out throughout England whereby a one-off unsedated FSIG is offered at age 55 [2].

BSSP FSIG is performed without sedation, although Entonox use is allowed. During the test, the endoscopist examines the colon as far as the participant's comfort and colonic preparation allows. Early data from BSSP have shown that one in three patients reported moderate or severe discomfort [3]. Optimal comfort is important not only to minimize harm to the participant, but to optimize participation in the programme [4].

\section{Water-assisted colonoscopy}

Reports on water-assisted colonoscopy (WAC) go back 30 years [5]. The technique involves water infusion during scope intubation, instead of air or Carbon dioxide $\left(\mathrm{CO}_{2}\right)$ insufflation. Two separate modalities have been described, although several variants are practiced: water immersion (WI) and water exchange (WE) [6]. WI involves water infusion to inflate the lumen during intubation, with aspiration performed predominantly on withdrawal of the scope. During WE, aspiration of infused water is predominantly during intubation; suction of residual air pouches or fecal residue also takes place in this phase [6]. The principle of both techniques is to minimize colonic distention and to wash the colon clean.

WAC, particularly the WE technique, may lead to improved patient comfort with less sedation [7-21]. Water may reduce friction between the endoscope and bowel wall, provide lubrication, and help keep the sigmoid straighter on scope insertion.

A recent randomized trial by Wang et al showed that benefits from WAC are still observed when the technique is applied solely during distal colon intubation, with the added advantage of a shorter overall procedure time compared to total-colon water intubation [22].

WAC may improve polyp detection by minimizing bowel distension, allowing subtle polyps to become more easily identifiable as they appear less "flattened." By achieving a cleaner colon, improved mucosal visualization can be seen on withdrawal. Longer intubation times described in some studies may also contribute to increased polyp detection [10,12,15-17, 21 25]. WAC may also aid completion of difficult [26] or previously incomplete procedures (due to angulations or redundant colons) [27]. Described therapeutic indications for WAC include endoscopic resolution of sigmoid volvulus and underwater polypectomy [28].

An increase in cecal intubation time (CIT) is a concern when performing WAC. Some studies point to significant prolongation [23] while others describe similar CITs [19] when operators become comfortable with the technique.
Studies show that WAC is safe, with no interference with fluid and electrolyte status [23].

\section{Patients and methods}

\section{WASh trial}

\section{Trial hypothesis}

We hypothesize that water-assisted sigmoidoscopy (WAS) reduces pain compared to $\mathrm{CO}_{2}$ insufflation, by minimizing bowel distension, allowing for a straighter passage of the scope through the colon with less looping.

\section{Primary and secondary outcomes}

The primary objective is to ascertain if procedural pain, assessed post-procedure and prior to discharge using a 4-point Likert scale ("None/Mild/Moderate/Severe"), is reduced in WAS compared to $\mathrm{CO}_{2}$ insufflation in people undergoing unsedated flexible sigmoidoscopy as part of BSSP. We chose to assess pain primarily by means of a Likert scale, as we feel it is more clinically meaningful for a study that may lead to practice changes.

The key secondary outcome is adenoma detection rate (ADR), the key performance indicator of sigmoidoscopy detecting meaningful colonic pathology.

The following criteria will be used to determine the overall success of the WAS technique; WAS will be considered successful if either:

- WAS comfort score (primary outcome) is significantly higher compared with $\mathrm{CO}_{2}$, with no indication ${ }^{1}$ of ADR (key secondary outcome) being inferior; or

- WAS comfort score (primary outcome) is not achieved (but comfort score not statistically significantly inferior with WAS) but key secondary outcome achieved (ADR improved with statistical significance)

A full list of secondary outcomes and how they will be measured is provided in $>$ Table 1 .

Stratification variables for the study are screening center, scope diameter (adult, pediatric) and history of hysterectomy. Subgroup analyses will be performed based on these variables.

\section{Trial design}

This is a multicenter, prospective, two-armed, randomized single blinded trial designed to evaluate performance of WAS in people referred for screening through the BSSP. Best efforts will be made to keep participants blinded from the treatment although we acknowledge this may not be possible in all instances.

We aim to randomize 1100 consecutive consenting participants to the trial with a $1: 1$ ratio between the two trial arms

\footnotetext{
$13 \%$ would be the difference with control ADR considered for a non-inferiority margin, in line with other studies. This would not reach statistical significance, but this level has been agreed with the NIHR RfPB panel as our predefined definition of "no indication of adenoma detection rate (ADR) being inferior".
} 
- Table 1 Detailed description of secondary outcome measures.

\begin{tabular}{|c|c|c|}
\hline Secondary & Rationale & Measure \\
\hline 1. ADR & $\begin{array}{l}\text { To assess whether WAS affects ADR in a positive } \\
\text { or negative manner }\end{array}$ & Percentage of procedures with adenomas detected \\
\hline $\begin{array}{l}\text { 2. Mean adenomas per } \\
\text { procedure (MAP) }\end{array}$ & To assess whether WAS affects MAP & Number of adenomas per procedure \\
\hline 3. Patient procedural pain & To assess whether WAS decreases pain & $\begin{array}{l}\text { Visual analogue scale (vas) }(0-100) \text { post-procedure, pre- } \\
\text { discharge }\end{array}$ \\
\hline 4. Patient experience & $\begin{array}{l}\text { To assess whether WAS leads to a better overall } \\
\text { patient experience. By utilising patient-derived } \\
\text { pain and experience assessments rather than } \\
\text { nurse or endoscopist-derived ones }\end{array}$ & $\begin{array}{l}\text { Post-discharge questionnaire assessing pain, embarrass- } \\
\text { ment, and willingness to repeat the procedure, expected } \\
\text { versus experienced pain, overall satisfaction and symptoms } \\
\text { post-procedure. To be filled the day after and posted back } \\
\text { to site. }\end{array}$ \\
\hline $\begin{array}{l}\text { 5. Sigmoidoscopy insertion } \\
\text { time (SIT) }\end{array}$ & $\begin{array}{l}\text { To assess whether WAS affects sit and, conse- } \\
\text { quently, overall procedure time }\end{array}$ & Duration of insertion \\
\hline $\begin{array}{l}\text { 6. Sigmoidoscopy with- } \\
\text { drawal time (SWT) }\end{array}$ & $\begin{array}{l}\text { To assess whether WAS affects SWT, and, conse- } \\
\text { quently, overall procedure time }\end{array}$ & Duration of withdrawal (in polyp-negative procedures) \\
\hline $\begin{array}{l}\text { 7. Maximum extent of } \\
\text { insertion }\end{array}$ & $\begin{array}{l}\text { To assess whether WAS leads to deeper scope } \\
\text { intubation }\end{array}$ & $\begin{array}{l}\text { Rectum, distal sigmoid, proximal sigmoid, distal descending, } \\
\text { proximal descending, splenic flexure, distal transverse; as } \\
\text { judged by endoscopist }\end{array}$ \\
\hline 8. Length of scope inserted & $\begin{array}{l}\text { To be used as surrogate for how straight/looped } \\
\text { the scope is (stratifying by segment extent). } \\
\text { Note - this is not a measure of depth of insertion }\end{array}$ & Length of scope inserted just prior to withdrawal \\
\hline 9. Entonox use & $\begin{array}{l}\text { To assess whether WAS affects need for Entonox } \\
\text { in a positive or negative manner }\end{array}$ & $\begin{array}{l}\text { Percentage of procedures where Entonox WAS used on de- } \\
\text { mand. This will not be applicable in procedures where parti- } \\
\text { cipants prefer to start their procedure with Entonox use }\end{array}$ \\
\hline 10. Quality of mucosal views & $\begin{array}{l}\text { To assess whether WAS influences quality of } \\
\text { mucosal views, as a consequence of the cleans- } \\
\text { ing effect of water irrigation }\end{array}$ & $\begin{array}{l}\text { Boston Bowel Preparation scale score for inspected seg- } \\
\text { ments, as assessed on withdrawal }\end{array}$ \\
\hline 11. Need for re-enema & To assess whether WAS affects re-enema rates & $\begin{array}{l}\text { Percentage of procedures where a second, through-the- } \\
\text { scope, enema WAS used. }\end{array}$ \\
\hline $\begin{array}{l}\text { 12. Need for external hand } \\
\text { pressures }\end{array}$ & $\begin{array}{l}\text { To assess whether WAS affects need for hand } \\
\text { pressure maneuvers during insertion; this can be } \\
\text { used as a surrogate for loop prevention }\end{array}$ & Percentage of procedures where hand pressure WAS required \\
\hline $\begin{array}{l}\text { 13. Need for patient position } \\
\text { changes }\end{array}$ & $\begin{array}{l}\text { To assess whether WAS affects need for position } \\
\text { changes during insertion }\end{array}$ & $\begin{array}{l}\text { Percentage of procedures where patient position change } \\
\text { WAS required }\end{array}$ \\
\hline $\begin{array}{l}\text { 14. Technique conversion } \\
\text { rates }\end{array}$ & $\begin{array}{l}\text { To assess frequency, reasons and caveats leading } \\
\text { endoscopists to switch between one of the two } \\
\text { study techniques. }\end{array}$ & Conversion rate from WAS to $\mathrm{CO}_{2}$, or $\mathrm{CO}_{2}$ to WAS technique \\
\hline $\begin{array}{l}\text { 15. Volume of water and } \mathrm{CO}_{2} \\
\text { used }\end{array}$ & $\begin{array}{l}\text { To calculate an average volume of water and } \mathrm{CO}_{2} \\
\text { needed for WAS and } \mathrm{CO}_{2} \text { techniques, for health } \\
\text { economics analysis reasons }\end{array}$ & $\begin{array}{l}\text { Volume of water (irrigator, syringe washes) and } \mathrm{CO}_{2} \text { (where } \\
\text { possible as per } \mathrm{CO}_{2} \text { pump specs) used in WAS and } \mathrm{CO}_{2} \text { proce- } \\
\text { dures }\end{array}$ \\
\hline 16. Scope looping & $\begin{array}{l}\text { To assess whether WAS leads to less looping, and } \\
\text { to correlate this to other study outcomes, e.g. } \\
\text { procedural pain. }\end{array}$ & Process described in relevant section of this protocol \\
\hline 17. Was learning curve & $\begin{array}{l}\text { To define the endoscopist learning curve of WAS } \\
\text { technique }\end{array}$ & $\begin{array}{l}\text { Capture endoscopists' reported confidence in performing } \\
\text { the procedure and their attitude towards the technique prior } \\
\text { to trial commencement, as well as performance (e. g. proce- } \\
\text { dure times, extent of insertion, conversion rates) as the study } \\
\text { progresses }\end{array}$ \\
\hline
\end{tabular}

ADR, adenoma detection rate; SIT, sigmoidoscopy insertion time; SWT, sigmoidoscopy withdrawal time 


\section{Assesed for eligibility $(n=5500)$}

Attending for Bowel Scope Screening Programme

$\begin{aligned} & \text { Excluded } 80 \% \\ & (\mathrm{n}=4400)\end{aligned}$
- Not meeting
inclusion criteria
$(\mathrm{n}=140)$
$(3 \%$ estimate)
- Declined/not
offered to
participate for time
reasons ( $\mathrm{n}=4260)$
assuming a final
uptake of $20 \%$

Randomised $(n=1100)$

Randomisation will be stratified for site, scope diameter (pediatric/adult) and hysterectomy (Male/ Female with/Female without).

$\downarrow$
Allocated to treatment
as usual $(\mathrm{n}=550)$

Received allocated

treatement $(n=522)$

Did not recieve allocated treatment $(n=28)$

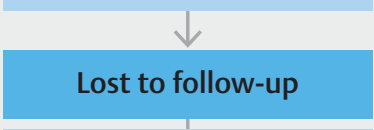

None anticipated based on study design $\checkmark$

Intention to treat
analysis $(\mathbf{n}=550)$

Per-protocol analysis $(n=522)$

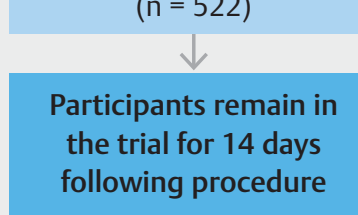

Clinical follow up as per routine practice

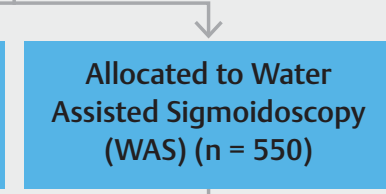

Received allocated treatement $(n=522)$

Did not recieve allocated treatment $(n=28)$

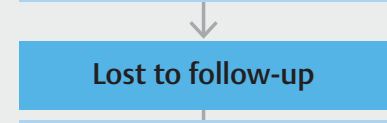

None anticipated based on study design

$\checkmark$

Intention to treat analysis $(n=550)$

Per-protocol analysis $(n=522)$

Participants remain in the trial for 14 days following procedure

Clinical follow up as per routine practice

Fig. 1 Patient flowchart.

( Fig. 1), within a timeframe of 18 months. Considering the quick patient turnaround times in BSSP lists, we have assumed an uptake rate of $20 \%$ per list. We anticipate that we will need to invite 5500 people to achieve this.

A patient trial information leaflet will be sent out to all potential participants confirming attendance for BSSP FSIG 2 weeks prior to their procedure. This will be done by the screening team and without involvement of the research team. No one except the screening team will have access to participantidentifiable information. Participants will be approached by a member of the research team on the day of the procedure and given the opportunity to discuss the trial. If they are willing to participate, fulfil the inclusion criteria and have no exclusion criteria, written consent will be obtained, and pre-procedure data will be collected.

\section{Inclusion criteria}

Patients attending for screening FSIG via BSSP that can give informed consent.

\section{Exclusion criteria}

1. Absolute contraindications to sigmoidoscopy

2. Participants lacking capacity to give informed consent for the procedure

3. Previous distal colonic/rectal resection.

4. Ongoing antithrombotic treatment (apart from aspirin, which is permitted).

\section{Withdrawal criteria}

Participants can withdraw at any time without giving reasons and without it effecting their further treatment.

\section{Setting/participating centers}

Participants will be recruited in five sites: four in the Northern Region (North Tees and Hartlepool Hospital, Northumbria Hospital, South Tyneside Hospital and County Durham and Darlington Hospitals) and St Mark's Hospital, London. A list of interested reserve sites is also in place should this is deemed necessary during recruitment.

Recruitment will begin at North Tees Hospital to allow testing of the protocol and refinement of the data collection process ( $\triangleright$ Fig. 2). If required, amendments will be performed and disseminated to participating sites as appropriate.

\section{Randomization}

Participants will be randomized in a 1:1 ratio to either $\mathrm{CO}_{2} \mathrm{FSIG}$ (Usual care) or WAS FSIG (Intervention). An electronic webbased randomisation system will be used. The randomisation process will use dynamic allocation [29] to ensure a consistent balance to the allocation ratio of $1: 1\left(\mathrm{CO}_{2}\right.$ : WAS) within stratification variables.

\section{Sample size and power}

The trial has been powered to detect a difference in the primary outcome of procedural pain. Data from a BSSP post-procedure patient survey suggested $38.2 \%$ felt moderate to severe pain from their FSIG. An improvement to $30 \%$ would be considered 


\section{Prior to trial start}

\begin{tabular}{|c|c|}
\hline $\begin{array}{l}\text { Completion of trial protocol } \\
\text { Patient information sheet, consent forms, GP letters and } \\
\text { patient invitation letters created }\end{array}$ & $\begin{array}{c}\text { Adverse Events and Serious Adverse Events completed } \\
\text { Standard operating procedure (SOP) and emergency } \\
\text { randomisation SOP completed }\end{array}$ \\
\hline & \\
\hline \multicolumn{2}{|c|}{ Training } \\
\hline $\begin{array}{l}\text { Bowel Scope Screening Programme (BSSP) endoscopists } \\
\text { identified and education package sent by e-mail }\end{array}$ & $\begin{array}{c}\text { Central training day with live demonstration of Water } \\
\text { assisted sigmoidoscopy (WAS) technique to research delivery } \\
\text { team }\end{array}$ \\
\hline \multicolumn{2}{|c|}{$\downarrow$} \\
\hline \multicolumn{2}{|c|}{ Trial set up } \\
\hline $\begin{array}{l}\text { Integrated Research approval system (IRAS) and Research } \\
\text { Ethics Committee (REC) submitted } \\
\text { REC and Health Research Authority (HRA) approval obtained }\end{array}$ & $\begin{array}{l}\text { SOA (Statement of Activity), SOE (Statement of Events) and } \\
\text { Local information pack sent to local Research \& Development } \\
\text { (R\&D) to ensure research nurse support/IT support/ } \\
\text { endoscopy unit set up appropriate }\end{array}$ \\
\hline \multicolumn{2}{|c|}{$\downarrow$} \\
\hline \multicolumn{2}{|c|}{ Site Initiation Visit } \\
\hline \multicolumn{2}{|c|}{ Recruitment of patients at sponsor site to ensure protocol can be delivered } \\
\hline \multicolumn{2}{|c|}{ Full Trial Roll Out } \\
\hline \multicolumn{2}{|c|}{ Site initiation Visit's (SIV's) monthly at other sites } \\
\hline \multicolumn{2}{|c|}{ Recruitment, data collection and monitoring by sponsor } \\
\hline \multicolumn{2}{|c|}{$\downarrow$} \\
\hline \multicolumn{2}{|c|}{ End of recruitment } \\
\hline \multicolumn{2}{|c|}{$\downarrow$} \\
\hline \multicolumn{2}{|c|}{ Analysis and write up } \\
\hline
\end{tabular}

Fig. 2 Trial flowchart.

clinically significant. Powered for this proportion of change with a $5 \%$ significance level and $80 \%$ power on a two-sided test, it is calculated that we would require 1048 participants to complete the trial. Assuming that some cases will need to be changed from WAS to $\mathrm{CO}_{2}$ and vice versa, we aim to recruit 1100 participants (550 per trial arm). It is estimated that $20 \%$ of invitees will finally agree to participate in the trial; therefore 5500 people will be invited.

\section{Endoscopist selection criteria}

Participating endoscopists will have a minimum experience of 300 BSSP procedures and an ADR greater than 6.8\%. All procedures will be performed by endoscopists trained in both $\mathrm{CO}_{2}$ and WAS techniques. We anticipate approximately 20 endoscopists will perform study procedures across all sites.

\section{Bowel Scope Screening Programme procedure}

As per standard BSSP practice, participants will self-administer a phosphate enema prior to the procedure. The procedure is performed without sedation. The participants have the option of using Entonox. Where available, magnetic endoscope ima- 
ging can be used. If the bowel preparation is deemed inadequate, a second, through-the-scope enema may be given at the endoscopist's discretion. The maximum extent of the test is splenic flexure. Comfort of the participant during the bowel scope screening procedure is paramount and the endoscopist will examine the colon as far as tolerance allows. Polyps up to $10 \mathrm{~mm}$ can be removed during the procedure provided it is felt feasible and safe to do so. BSSP conversion-to-colonoscopy guidelines will be followed, as per standard practice.

\section{Water-assisted sigmoidoscopy technique}

The primary concept of the WAS technique employed in our trial is to keep the lumen as collapsed as possible, thereby concertinaing the sigmoid colon, reducing the tendency for looping and resulting in a straighter and "shorter scope" passage to descending colon. WAS is a different technique than WAC and we will explain the principles of this in the following paragraph.

The WAS technique consists of turning off the $\mathrm{CO}_{2}$ pump shortly after scope insertion through the anus, as soon as the rectal ampulla has been visualized. Minimal water volume is then infused to achieve adequate luminal views as the scope advances. Pockets of air encountered during intubation through the sigmoid colon should be actively suctioned. We emphasise use of pulses of water to achieve adequate luminal views to advance the scope, without necessarily aiming to distend/fill the lumen with water. Suctioning of water/fecal residue is performed as needed. The WAS technique is ideally performed without any gas insufflation; however, one or two short blasts of $\mathrm{CO}_{2}$ are permitted, at the discretion of the endoscopist. Where possible, insufflated gas should then be suctioned as soon as feasible, adhering to the principle of keeping the colon as collapsed as possible.

At the maximal point of intubation, the $\mathrm{CO}_{2}$ pump is turned back on. $\mathrm{CO}_{2}$ insufflation at this point often "pushes" water/fecal residue proximally, allowing deeper views. Scope withdrawal is done as per standard practice using $\mathrm{CO}_{2} /$ water as needed.

\section{Analysis of sigmoid looping}

We plan to use an expert Delphi consensus to identify and agree upon important components of sigmoid looping in relation to pain that are measurable on magnetic endoscope imaging (MEI). Two assessors will then apply the components to a select number of test $\mathrm{MEI}$ videos to measure agreement for each component. The agreed components can then be applied to $\mathrm{MEI}$ videos, which have been recorded (when recording equipment are available) from the WASh trial. This will allow us to correlate loop components with pain scores and study looping patterns for each arm of the trial.

\section{Training}

An initial education package will be sent to endoscopists at participating sites. This will include details of the WASh trial, current literature on WAC, a description of the specific technique of WAS, a video demonstrating WAS, baseline questionnaire and a non-mandatory training log.
Participating endoscopists will be invited to attend a training event which will comprise an educational session to review details of the WASh trial, current literature on WAC, breakdown of the WAS technique (including further videos) and live demonstration of the technique. Endoscopists will be encouraged to practise WAS technique prior to attending the training day. Concerns that may have arisen during the above "practice" period will also be addressed on the event day.

Participating endoscopists will be contacted monthly during their training period, to monitor progress made as well as to express any concerns regarding the trial and the WAS technique. Additional one-to-one training sessions will be offered upon request, before and during trial conduct, to address any issues regarding the WAS technique.

Prior to participating in the WASh trial, endoscopists will be required to have performed at least 20 WAS cases at their sites, and confirm that they feel fully competent in performing the WAS technique. We aim to observe rea- life procedures or a video recording of each endoscopist performing WAS.

During the trial, endoscopists will be contacted every 2 months to give them an opportunity to discuss WAS technique and to access further training/advice if required.

\section{Adverse events}

Risk of Adverse Events (AEs) with WAC is thought to be the same as with standard colonoscopy. We assume this will also be the case for WAS.

The trial will measure AEs, which will be recorded in the BSSP database and on case report forms. AEs will be recorded retrospectively for the 14-day period from the day of the sigmoidoscopy, or until withdrawal.

We acknowledge that the information sent in advance to people invited for Bowel Scope could induce anxiety and adversely affect attendance for screening. To address this, we will capture the "Did Not Attend" (DNA)/cancellation rates and task the Data Monitoring and Ethics committee (DMEC) with monitoring this.

\section{Assessment and follow-up}

Clinical follow-up will be as per routine clinical practice for each participating unit. All participants with identified polyps will have their post-BSSP FSIG management plan as usual [2]. Eligible, consented participants will remain in the trial for 14 days following their procedure, for AE identification purposes. No additional visits are required. The timescale for any outpatient appointments and subsequent care will be unaltered by participation in the trial.

\section{Data analysis}

The study will be analyzed on an intention-to-treat basis and a full statistical analysis plan incorporating a health economics analysis plan will be written and agreed to prior to completion of data collection.

The primary outcome of pain will be analyzed using logistic regression to assess differences between the two treatment groups. Two models will be created, the first where participants reported either moderate or severe pain and the second for 
those that only reported severe pain. Stratification variables along with gender, endoscopist and any other variables deemed appropriate will be included within the regression models.

ADR will be analyzed using logistic regression models. The mean ADR rate for each of the groups will be calculated and if the difference between groups is not statistically significant but the percentages are within $3 \%$ of each other, that will be considered a suitable conclusion for the trial, as described in the aims section.

The Visual Analogue Scale (VAS) pain score, collected from participants on the day of the procedure, will be assessed using analysis of covariance (ANCOVA) model adjusting for the important variables as defined in the primary outcome regression model. Other participant-reported outcomes such as embarrassment and overall satisfaction that are collected the next day from the participant will be analyzed in the same way.

Further analysis of secondary outcomes will be completed with continuous variables analyzed using ANCOVA models and categorical variables being analyzed using logistic regression models. Sensitivity analysis will be completed using a dichotomy of the Likert scale using none, mild and moderate versus severe. Due to the nature of the data collection it is anticipated that missing data will be minimal, however, assessment of missing data will be made and appropriate multiple imputation techniques will be employed where necessary.

Where appropriate, subgroup analysis will be considered for certain cohorts that have been deemed significant from the models described above, including previous hysterectomy, irritable bowel syndrome, and diverticulosis, as well as depth of scope insertion (segment and length of scope), scope diameter (adult vs paediatric) and model (e.g. Olympus 240/260/290) and individual endoscopists. Outcomes will be assessed on an intention-to-treat basis and subsequently on a per-protocol basis as applicable to assess the sensitivity of the results.

A full statistical analysis plan will be written and ratified by the trial steering committee (TSC) and DMEC. Data and all appropriate documentation will be stored for a minimum of 15 years after study completion, including the follow-up period, in compliance with regulatory authority archiving requirements.

\section{Health economic analysis}

The trial includes a health economics component, incorporating

- Cost-effectiveness analyzes of WAS versus $\mathrm{CO}_{2}$ intubation

- A discrete choice experiment (DCE) to explore relative preferences of participants for individual attributes of sigmoidoscopy (pain, likelihood of missing an abnormality, procedure time, bowel cleansing, risk of serious complication e. g. bleeding or perforation), relative importance of different attributes, and how participants make trade-offs between attributes. The DCE survey will be completed by participants the day after their procedure

\section{Cost-effectiveness}

Cost-effectiveness analyses [30,31] will be undertaken to compare relative costs and effects of WAS versus $\mathrm{CO}_{2}$ techniques, using procedural pain score and number of adenomas detected as the measures of effect. The incremental cost-effectiveness ratio (ICER) point estimate [30-32] will be calculated, expressed in cost per unit difference in pain and cost per unit difference in adenomas detected.

Duration of procedures and staff levels/bands working in the room will be captured to facilitate cost analysis of the impact, if any, of the trial technique to costs in terms of time.

We will present a cost-effectiveness plan to illustrate scatter plots of the joint distribution of the pairs of costs and effects. A cost-effectiveness acceptability curve (CEAC) will be used to show the probability of WAS being cost-effective compared to $\mathrm{CO}_{2}$ over a range of cost-effectiveness thresholds. These thresholds will present the maximum acceptable ceiling ratio a decision-maker is willing to pay for one unit gained in effect $[31,33]$. Sensitivity analysis, where appropriate, will be performed to explore the impact of any assumptions made on results. We will conduct subgroup analysis on the basis of gender or age, where appropriate.

\section{Discrete choice experiment (DCE)}

As there are no comparative thresholds for cost-effectiveness using pain and adenomas detected as outcome measures, a discrete choice experiment (DCE) will be employed to explore: (1) the relative preferences of participants for individual attributes of sigmoidoscopy (pain, likelihood of missing an abnormality, procedure time, bowel cleansing, risk of serious complication e. g. bleeding perforation); (2) the relative importance of different attributes; and (3) how participants make trade-offs between attributes $[34,35]$. DCE data will be analysed using logistic regression techniques.

\section{Data monitoring and ethics}

The DMEC will include an independent chair, independent clinician, independent statistician, and WASh study statistician. The DMEC will review trial status during study conduct, including accumulated outcome data and AEs, and will also comment on the statistical analysis plan when convening.

\section{Trial steering committee}

The Trial Steering Committee will comprise an independent chair, two independent clinicians, an independent health economist, independent statistician, representation from sponsor site, WASh study statistician and health economist, chief investigator for the WASh study and a patient and public involvement representative. The role of the Trial Steering Committee is to supervise the trial to ensure that it is conducted to the rigorous standard.

\section{Trial period}

The trial will last 30 months. Recruitment period will extend from December 2018 to June 2019. 


\section{Protocol version}

WASh BSSP Protocol V4.0, 8th Nov 2017.

\section{Ethical considerations}

Ethics approval gained from the North East Ethics committee who gave a favourable outcome.

\section{Sponsor}

North Tees and Hartlepool NHS Foundation Trust will be the sponsor for this trial.

\section{Discussion}

This will be the first trial to investigate the effects of WAS in a screening setting. If the trial shows that WAS either reduces pain or increases ADR, this may result in a NHS Bowel Cancer Screening Programme practice change to implement WAS, particularly if it proves to be cost-neutral. This will directly impact on the 256,000 people a year who will undergo bowel scope by 2020. We anticipate WAS could be introduced into non-screening endoscopic practice as the skills are transferable and the benefits are likely to be generalizable.

\section{Competing interests}

None

\section{References}

[1] Atkin WS, Edwards R, Kralj-Hans I et al. Once-only flexible sigmoidoscopy screening in prevention of colorectal cancer: a multicentre randomised controlled trial. Lancet 2010; 375: 1624-1633

[2] Bevan R, Rubin G, Sofianopoulou E et al. Implementing a national flexible sigmoidoscopy screening program: results of the English early pilot. Endoscopy 2015; 47: 225-231

[3] Von Wagner C. Patient Experience of Bowel Cancer Screening: What are we measuring? Liverpool, United Kingdom: British Society of Gastroenterology; 2016

[4] McLachlan SA, Clements A, Austoker J. Patients' experiences and reported barriers to colonoscopy in the screening context-a systematic review of the literature. Patient Educ Couns 2012; 86: 137-146

[5] Falchuk ZM, Griffin PH. A technique to facilitate colonoscopy in areas of severe diverticular disease. N Engl J Med 1984; 310: 598

[6] Leung FW. Water-aided colonoscopy. Gastroenterol Clin North Am 2013; 42: $507-519$

[7] Leung FW, Harker JO, Jackson G et al. A proof-of-principle, prospective, randomized, controlled trial demonstrating improved outcomes in scheduled unsedated colonoscopy by the water method. Gastrointest Endosc 2010; 72: 693 - 700

[8] Leung J, Mann S, Siao-Salera R et al. A randomized, controlled trial to confirm the beneficial effects of the water method on U.S. veterans undergoing colonoscopy with the option of on-demand sedation. Gastrointest Endosc 2011; 73: $103-110$

[9] Pohl J, Messer I, Behrens A et al. Water infusion for cecal intubation increases patient tolerance, but does not improve intubation of unsedated colonoscopies. Clin Gastroenterol Hepatol 2011; 9: 1039 1043.e1031
[10] Ramirez FC, Leung FW. A head-to-head comparison of the water vs. air method in patients undergoing screening colonoscopy. J Interv Gastroenterol 2011; 1: 130-135

[11] Falt P, Liberda M, Smajstrla $V$ et al. Combination of water immersion and carbon dioxide insufflation for minimal sedation colonoscopy: a prospective, randomized, single-center trial. Eur J Gastroenterol Hepatol 2012; 24: $971-977$

[12] Leung FW, Amato A, Ell C et al. Water-aided colonoscopy: a systematic review. Gastrointest Endosc 2012; 76: 657-666

[13] Amato A, Radaelli F, Paggi S et al. Carbon dioxide insufflation or warm-water infusion versus standard air insufflation for unsedated colonoscopy: a randomized controlled trial. Dis Colon Rectum 2013; 56: $511-518$

[14] Falt P, Smajstrla V, Fojtik P et al. Cap-assisted water immersion for minimal sedation colonoscopy: prospective, randomized, single-center trial. Dig Endosc 2013; 25: 434-439

[15] Cadoni S, Gallittu P, Sanna S et al. A two-center randomized controlled trial of water-aided colonoscopy versus air insufflation colonoscopy. Endoscopy 2014; 46: 212-218

[16] Hsieh YH, Koo M, Leung FW. A patient-blinded randomized, controlled trial comparing air insufflation, water immersion, and water exchange during minimally sedated colonoscopy. Am J Gastroenterol 2014; 109: 1390 - 1400

[17] Anderson JC. Water-aided colonoscopy. Gastrointest Endosc Clin N Am 2015; 25: 211-226

[18] Garborg K, Kaminski MF, Lindenburger W et al. Water exchange versus carbon dioxide insufflation in unsedated colonoscopy: a multicenter randomized controlled trial. Endoscopy 2015; 47: 192 - 199

[19] Cadoni S, Falt P, Gallittu P et al. Water exchange is the least painful colonoscope insertion technique and increases completion of unsedated colonoscopy. Clin Gastroenterol Hepatol 2015; 13: 1972 - 1980

[20] Cadoni S, Sanna S, Gallittu P et al. A randomized, controlled trial comparing real-time insertion pain during colonoscopy confirmed water exchange to be superior to water immersion in enhancing patient comfort. Gastrointest Endosc 2015; 81: 557-566

[21] UEG Week 2015 Poster Presentations. United European gastroenterology journal 2015; 3: 146-687

[22] Wang X, Luo H, Xiang Y et al. Left-colon water exchange preserves the benefits of whole colon water exchange at reduced cecal intubation time conferring significant advantage in diagnostic colonoscopy - a prospective, randomized controlled trial. Scand J Gastroenterol 2015; 50: $916-923$

[23] Rex DK. Water exchange vs. water immersion during colonoscope insertion. Am J Gastroenterol 2014; 109: 1401 - 1403

[24] Leung F, Harker J, Leung J et al. Removal of infused water predominantly during insertion (water exchange) is consistently associated with a greater reduction of pain score - review of randomized controlled trials (RCTs) of water method colonoscopy. J Interv Gastroenterol 2011; 1: 114-120

[25] Hafner S, Zolk K, Radaelli F et al. Water infusion versus air insufflation for colonoscopy. Cochrane Database Syst Rev 2015: doi:10.1002/ 14651858.CD009863.pub2

[26] Luo H, Zhang L, Liu X et al. Water exchange enhanced cecal intubation in potentially difficult colonoscopy. Unsedated patients with prior abdominal or pelvic surgery: a prospective, randomized, controlled trial. Gastrointest Endosc 2013; 77: 767-773

[27] Vemulapalli KC, Rex DK. Water immersion simplifies cecal intubation in patients with redundant colons and previous incomplete colonoscopies. Gastrointest Endosc 2012; 76: 812-817

[28] Sugimoto S, Mizukami T. Diagnostic and therapeutic applications of water-immersion colonoscopy. World J Gastroenterol: WJG 2015; 21: $6451-6459$ 
[29] Russell D, Hoare ZS, Whitaker R et al. Generalized method for adaptive randomization in clinical trials. Stat Med 2011; 30: 922 - 934

[30] Glick H, Doshi JA, Sonnad SS et al. Economic evaluation in clinical trials. Oxford: Oxford University Press; 2015

[31] Drummond MF, Sculpher M], Claxton K et al. Methods for the economic evaluation of health care programmes. Fourth edition. Oxford: Oxford University Press; 2017

[32] Morris S, Devlin N, Parkin D. Economic analysis in health care. Chichester: John Wiley \& Sons, Ltd; 2007
[33] Fenwick E, Marshall DA, Levy AR et al. Using and interpreting cost-effectiveness acceptability curves: an example using data from a trial of management strategies for atrial fibrillation. BMC Health Serv Res 2006; 6: 52

[34] Yeo ST, Edwards RT, Fargher EA et al. Preferences of people with diabetes for diabetic retinopathy screening: a discrete choice experiment. Diabet Med 2012; 29: 869-877

[35] Ryan M, Scott DA, Reeves C et al. Eliciting public preferences for healthcare: a systematic review of techniques. Health Technol Assess 2001; 5: 1-186 